\title{
The Role of Preoperative Chest CAT Scan in Cardiac Surgical Patients
}

Marie-José de Hartog-Dikhoff ${ }^{1 *}$, Liu-Noor Overweg ${ }^{2}$ and Willem Stooker ${ }^{1}$

${ }^{1}$ Department of Cardiothoracic Surgery, OLVG Amsterdam, The Netherlands

${ }^{2}$ University of Amsterdam, Medical School, The Netherlands

\begin{abstract}
Introduction: Arteriosclerosis of the ascending aorta is increasingly encountered in cardiac surgical patients as patients accepted for cardiac surgery get older. We evaluated the effect of introducing preoperative Chest CAT scan in the preoperative work-up for cardiac surgery concerning the change in strategy.

Materials and methods: From May 1st 2015 until October 1st 2015 all patients of 70 years and older as well as younger patients with peripheral vascular disease presented in the heart team and accepted for surgery received a preoperative Chest CAT scan. We evaluated in which patients the initially established treatment was changed. Furthermore we investigated which patients had a stroke after surgery.

Results: 583 patients were discussed in the Heart Team during the study period. 290 patients were accepted for surgery. 195 patients fitted the criteria for a CAT scan. In this group in 18 patients $(9.2 \%)$ the treatment was changed because of severe calcification of the ascending aorta or peripheral cannulation site. None of the 272 patients who underwent surgery had a stroke.

Conclusion: Preoperative Chest Cat scan gives essential information concerning the calcification of the ascending aorta and should be recommended in the preoperative work-up at least in elderly patients and patients with peripheral vascular disease.
\end{abstract}

Keywords: Preoperative; Cardiac surgery; CAT scan; Ascending aorta; Calcification

\section{Introduction}

As patients referred for cardiac surgery are getting older and older, more atherosclerotic problems are encountered. Especially arteriosclerosis of the ascending aorta offers a technical challenge for cardiothoracic surgeons concerning canulation and surgical procedures, e.g., proximal anastomosis of a vein graft or aortic valve surgery. Incidentally a porcelain aorta is found after sternotomy prohibiting further operation at all. In our opinion it's important to be informed about the extensiveness of the ascending aortic calcification to determine the operative strategy and most appropriate canulation technique. In our department we use epi-aortic ultrasound scanning in every patient for imaging of the ascending aorta before canulation. However, this is only possible after sternotomy. It is desirable to be well informed before the operation. Preoperative ultrasound and coronary artery angiography seldom show the extensiveness of the calcification in the ascending aorta. Severe calcification is especially encountered in older patients and in patients with known peripheral vascular disease. To make sure to be well informed about the extensiveness of the calcification of the ascending aorta we decided to implement preoperative Chest CAT scanning in every patient accepted for cardiac surgery from 70 years and older and in younger patients with peripheral vascular disease.

\section{Materials and Methods}

From May 1st until October 1st 2015 all patients referred to the Heart Team of our department were included in this study. The Heart Team consists of at least one cardiothoracic surgeon, one interventional cardiologist and one imaging cardiologist. All patients accepted for surgery of 70 years and older and younger patients with peripheral vascular disease had a preoperative chest CAT scan before surgery. If possible we chose for non-contrast Chest CAT scanning in order to prevent contrast nephropathy in the abovementioned group of patients in which often impaired renal function is found. An example of the findings in one of our patients is shown in Figure 1. All patients for minimal invasive mitral valve surgery, re-do procedures and minimal access aortic valve surgery had a CAT scan with contrast. We evaluated the effect of the implementation of preoperative Chest CAT scanning on operative strategy and the incidence of stroke. If the CAT scan showed

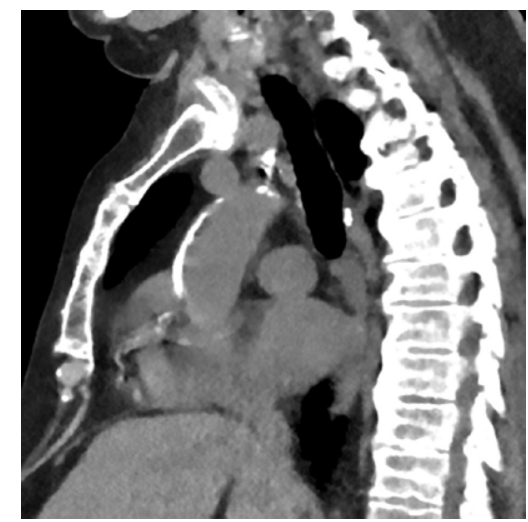

Figure 1: CAT scan without contrast showing severe calcification of ascending aorta.

*Corresponding author: MJ de Hartog-Dikhoff, MD, Department of Cardiothoracic Surgery, OLVG Amsterdam, The Netherlands, Tel: +31205993669; Fax: +31205993675; E-mail: M.Dikhoff@olvg.nl

Received August 16, 2017; Accepted August 25, 2017; Published August 29 2017

Citation: de Hartog-Dikhoff MJ, Overweg LN, Stooker W (2017) The Role of Preoperative Chest CAT Scan in Cardiac Surgical Patients. J Vasc Med Surg 5: 332. doi: 10.4172/2329-6925.1000332

Copyright: @ 2017 de Hartog-Dikhoff MJ, et al. This is an open-access article distributed under the terms of the Creative Commons Attribution License, which permits unrestricted use, distribution, and reproduction in any medium, provided the original author and source are credited. 
severe calcification of the ascending aorta making surgery impossible or an unacceptable high risk, the patient was again discussed in the Heart Team to evaluate the patient for an alternative treatment. During the postoperative stay in the hospital it was determined whether or not the patient had a stroke. The study was approved by the medical ethical committee.

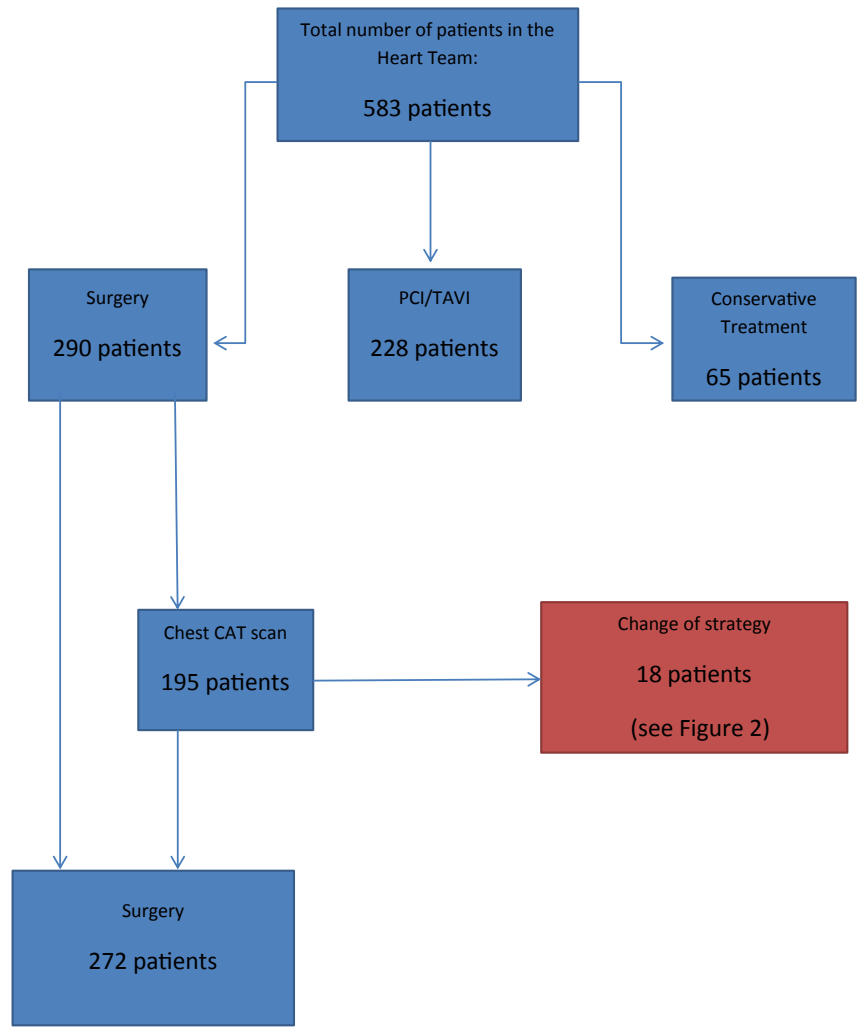

Figure 2: Flow chart detailing patients referred to the heart team.

\section{Results}

The results are shown in Figure 2. In total 583 patients were discussed in the Heart Team in the abovementioned period. None of the patients in the study group died. 290 patients were accepted for surgery. From this group in 95 cases CAT scanning was not performed on behalf of our criteria (patient younger than 70 years of age and no peripheral vascular disease). In 195 a CAT scan was made and in 18 (9.2\%) patients this gave rise to change the strategy. These cases are shown in Table 1 . None of the patients who underwent surgery had a stroke.

\section{Discussion}

Patients referred for cardiac surgery are getting older and older. Due to increasing vitality of octogenarians (and incidentally even nonagenarians), and improved technical possibilities, elderly patients are accepted for cardiac surgery. For many indications preoperative imaging of the ascending and descending aorta is used as a standard procedure in the work-up, e.g. in redo procedures, aortic and/ or aortic valve surgery, and minimal invasive procedures. In firsttime, conventional cardiac surgery however this is not customary. As arteriosclerosis is increasing with advanced age it is very important to be aware of limitations to surgical possibilities due to a calcified aorta, especially the ascending aorta, preferably before the operation. Unforeseen porcelain aorta is a disastrous discovery after sternotomy. This is also true for an unexpected dilated ascending aorta. Perioperative stroke due to embolization during surgery is an often disabling complication after cardiac surgery and of very high concern especially for the patients and their family but also for clinicians. Epi-aortic scanning gives information on the calcification and also is highly specific for identification of soft plaques, but is only possible after sternotomy. It has the advantage that also soft plaques are visible. A-view enhanced transesophageal echocardiography is only possible after induction of anesthesia and needs special skills from the anesthesiologists to place the saline filled balloon in the trachea [1-3].

Preoperative Chest CAT scan is a very convenient way to get

\begin{tabular}{|c|c|c|c|}
\hline S.No & $\mathrm{m} / \mathrm{f}$ & age & Initial proposed treatment \\
\hline 1 & M & 75 & AVR + CABG \\
\hline 2 & V & 72 & CABG \\
\hline 3 & M & 74 & CABG \\
\hline 4 & M & 80 & AVR \\
\hline 5 & M & 80 & AVR \\
\hline 6 & V & 81 & $A V R+C A B G$ \\
\hline 7 & M & 77 & AVR \\
\hline 8 & $M$ & 72 & CABG \\
\hline 9 & M & 83 & AVR \\
\hline 10 & V & 80 & AVR \\
\hline $11^{*}$ & V & 62 & Minimal invasive MPL \\
\hline 12 & M & 89 & CABG \\
\hline 13 & M & 70 & AVR \\
\hline 14 & $M$ & 74 & Re-CABG \\
\hline 15 & M & 65 & CABG \\
\hline 16 & V & 53 & AKV \\
\hline 17 & $M$ & 76 & Minimal invasive MPL \\
\hline 18 & M & 64 & CABG \\
\hline
\end{tabular}

\begin{tabular}{|l|l|}
\hline CAT scan findings & Change of strategy \\
\hline Severe calcification ascending aorta & PCI + TAVI \\
\hline Severe calcification ascending aorta & OPCAB \\
\hline Severe calcification ascending aorta & PCI \\
\hline Severe calcification ascending aorta & TAVI \\
\hline Severe calcification ascending aorta & TAVI \\
\hline Severe calcification ascending aorta & PCI + TAVI \\
\hline Severe calcification ascending aorta & TAVI \\
\hline Severe calcification ascending aorta & OPCAB \\
\hline Severe calcification ascending aorta & TAVI \\
\hline Severe calcification ascending aorta & TAVI \\
\hline Severe peripheral artery disease & Conventional MPL \\
\hline Severe calcification ascending aorta & OPCAB \\
\hline Severe calcification ascending aorta & TAVI \\
\hline LIMA adherent to sternum & PCI \\
\hline Severe calcification ascending aorta & OPCAB \\
\hline Severe calcification ascending aorta & TAVI \\
\hline Severe peripheral artery disease & Conventional MPL \\
\hline Severe calcification ascending aorta & PCI \\
\hline
\end{tabular}

m/f: Male/Female; AVR: Aortic Valve Replacement; CABG: Coronary Artery Bypass Grafting; PCl: Percutaneous Coronary Artery Intervention; TAVI: Transfemoral Aortic Valve Implantation; MPL: Mitral Valve Plasty; OPCAB: Off Pump Coronary Artery Bypass; Re-CABG: Redo Coronary Artery Bypass Grafting

${ }^{*}$ CAT scan with contrast was made of complete aorta and iliac and femoral arteries.

Table 1: Change of strategy. 
information of the aorta not during surgery but before the operation is planned. It is easily accessible, not expensive, and the radiation exposure is limited. Contrast is not necessary in the majority of cases to get a good image of the calcifications, so impairment of renal function is not relevant. The information is obtained before surgery, making it possible to discuss change of strategy with the patient and inform the patient of a possible higher complication rate like e.g. stroke. The stroke rate in our study group is zero, however, as our group is relatively small further investigation is necessary. Other groups confirmed the beneficial effect of introducing preoperative CAT scanning on perioperative stroke rates. In conclusion we strongly recommend preoperative non-contrast Chest CAT scan in patients with peripheral vascular disease and or patients of 70 years and older. In case of redo surgery with patent bypass grafts and in the judgment of peripheral arteries in minimal invasive procedures CAT scan with contrast is to be preferred.

\section{References}

1. Lee R, Matsutani N, Polimenakos AC, Levers LC, Lee M, et al. (2007) Preoperative noncontrast chest computed tomography identifies potential aortic emboli. Ann Thorac Surg 84: 38-41.

2. Nishi H, Mitsuno M, Tanaka H, Ryomoto M, Fukui S, et al. (2010) Who needs preoperative routine chest computed tomography for prevention of stroke in cardiac surgery? Interact Cardiovasc Thorac Surg 11: 30-33.

3. Patil TA, Nierich A (2016) Transesophageal echocardiography evaluation of the Thoracic Aorta. Ann Card. Anesth 19: S44-55. 